\title{
Temporal dynamics of shallow subtidal meiobenthos from a beach in Tenerife (Canary Islands, northeast Atlantic Ocean)
}

\author{
Rodrigo Riera ${ }^{1 *}$, Jorge Núñez ${ }^{2}$, María del Carmen Brito ${ }^{2}$ \\ ${ }^{1}$ Centro de Investigaciones Medioambientales del Atlántico (CIMA SL), Arzobispo Elías Yanes 44, 38206 \\ La Laguna, Tenerife, Canary Islands, Spain \\ ${ }^{2}$ Benthos Laboratory, Department of Animal Biology, Faculty of Biology, University of La Laguna, 38206 \\ La Laguna, Tenerife, Canary Islands, Spain
}

Received 23 January 2012; accepted 11 January 2013

CThe Chinese Society of Oceanography and Springer-Verlag Berlin Heidelberg 2013

\begin{abstract}
A shallow subtidal (3 m deep) meiofaunal assemblage in Los Abrigos Bay, Tenerife, Canary Islands was sampled during May 2000-April 2001. The main aims were to (1) find temporal variations in meiofaunal assemblage structure and overall abundance, as well as in the most abundant meiofaunal species throughout the study period, and (2) identify environmental variables (sedimentary composition, organic matter content, and total nitrogen) that better explain meiofaunal assemblage structure during the study year. The most abundant species were the nematodes Siphonolaimus sp. 2 and Catanema sp., which represented $46.2 \%$ of the overall meiofaunal abundances and varied significantly throughout the study duration. Overall meiofaunal abundance and the most abundant taxonomic groups (nematodes, copepods, and oligochaetes) showed significant temporal variations during the study period. Nematodes overwhelmingly dominated during the study period, ranging from $78 \%$ in May to $97.34 \%$ in February. Multivariate analyses showed seasonality in meiofaunal community structure during the study year, with the lowest abundances in May. Key words: Meiofauna, assemblage structure, subtidal, Canary Islands, Atlantic Ocean
\end{abstract}

Citation: Rodrigo Riera, Jorge Núñez, María del Carmen Brito. 2013. Temporal dynamics of shallow subtidal meiobenthos from a beach in Tenerife (Canary Islands, northeast Atlantic Ocean). Acta Oceanologica Sinica, 32(8): 44-54, doi: 10.1007/s13131-0130340-2

\section{Introduction}

Seasonal variation in animal populations is often quite predictable. In benthic communities, temporal changes are generally more pronounced in the intertidal than in deeper waters. A plethora of abiotic and biotic variables may account for temporal fluctuations in benthic animal assemblages (Ólafsson and Elmgren, 1997). Temporal distribution of meiofauna (mobile metazoan benthic invertebrates that pass through a $0.5 \mathrm{~mm}$ mesh sieve but are retained on a 0.063 or $0.042 \mathrm{~m}$ $\mathrm{m}$ mesh sieve) is highly variable and scale-dependent (Higgins and Thiel, 1988). Several meiobenthic studies in subtidal areas have failed to detect temporal oscillations in meiofaunal assemblages (Juario, 1975; Warwick and Buchanan, 1971; Boucher, 1980). However, other studies have observed significant seasonality in subtidal meiofauna assemblages (Rudnick et al., 1985, Ólafsson and Elmgren, 1997) throughout a study year.

In recent years, many ecological studies on meiobenthic communities have been carried out in the Canary archipelago (Riera, 2004; Brito et al., 2005). Brito et al. (2005) analysed seasonal variations of "large" meiofaunal/"small" macrofaunal polychaetes $(<0.1 \mathrm{~mm})$ from a Cymodocea nodosa seagrass meadow in Tenerife. However, "real" subtidal meiofauna (0.063-1 mm long) have not received attention and so, more baseline data describing species diversity and community structure are needed to further study the importance of meiofauna in shallow subtidal sandy systems.

In the present study, we investigated patterns in the as- semblage structure and abundance of meiofauna inhabiting a shallow subtidal site at the Canary Islands through an annual cycle. The main aims were to (1) determine if patterns of assemblage structure or meiofaunal abundances followed any temporal trend, and (2) identify if studied environmental variables (grain size, organic matter, and nitrogen) affected meiofauna assemblage structure throughout the study period.

\section{Materials and methods}

\subsection{Study area}

This study was conducted from May 2000 to April 2001 at Los Abrigos del Porís Bay, a locality on the southeast coast of Tenerife (Canary Islands, NE Atlantic Ocean, $\left(28^{\circ} 08^{\prime} 58^{\prime \prime} \mathrm{N}\right.$, $16^{\circ} 42^{\prime} 54^{\prime \prime} \mathrm{W}$ ) (Fig. 1). The study site is located in a sheltered bay (classified as ultra-dissipative, sensu Short, 1999). Sandy substrates are characterized by volcanic sands with a low percentage of carbonates, and dominated by the presence of medium and fine sands.

Sediment samples were collected monthly by SCUBA divers at one shallow subtidal station ( $3 \mathrm{~m}$ deep). Sediment cores $\left(10 \mathrm{~cm}^{2}\right)$ were pushed into the sediment to a depth of 30 $\mathrm{cm}$. Five replicates were collected for faunistic determinations and one for analysis of sedimentary variables (grain size, organic matter, and nitrogen).

\subsection{Analysis of sedimentological variables}

To assess grain size composition of the analysed sediment,

$\overline{{ }^{*} \text { Corresponding author, E-mail: rodrigo@cimacanarias.com }}$ 


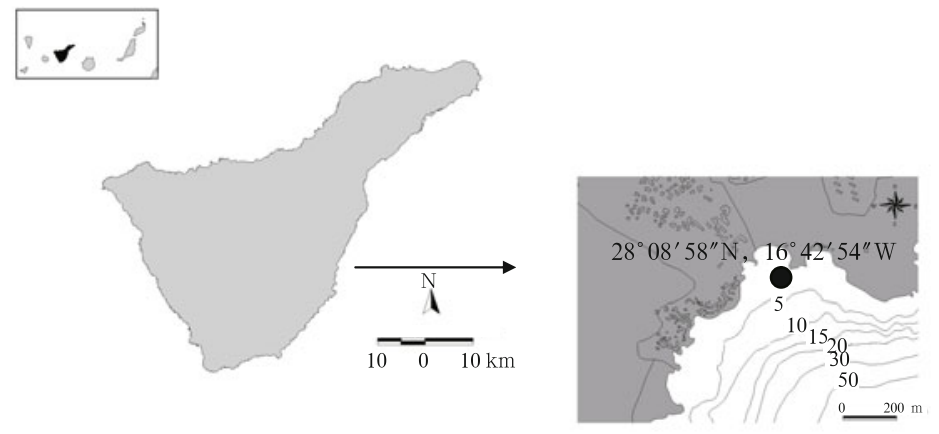

Fig.1. Map of the study area showing sampling location.

$100 \mathrm{~g}$ sediment from each month was oven dried at $105^{\circ} \mathrm{C}$, passed through a graded series $(2,1,0.5,0.25,0.125$, and 0.063 $\mathrm{mm}$ ) of sieves, and then weighed (Buchanan, 1984). Total nitrogen was determined by the Kjeldahl method (Bradstreet, 1965).

\subsection{Analysis of meiofauna}

Samples were preserved in $10 \%$ seawater-buffered formaldehyde solution and decanted through $500 \mu \mathrm{m}$ and 63 $\mu \mathrm{m}$ mesh sieves. The fraction remaining on the $63 \mu \mathrm{m}$ mesh sieve was separated into different taxonomic groups under a binocular microscope and preserved in $70 \%$ ethanol. The extraction method followed procedures of Somerfield and Warwick (1996). Meiofaunal specimens were mounted in jelly glycerine and examined using a LEICA DMLB microscope equipped with Nomarski interference contrast and identified to species level, whenever possible. All meiofaunal specimens were identified using the microscope and subsamples of 200 individuals were used for nematodes (Somerfield and Warwick, 1996). Certain taxonomic groups (i.e., harpacticoid copepods, turbellarians) that needed dissection or taxonomic characters of internal anatomy were determined by their body shape in putative species. Meiofaunal abundances were expressed as a number of individuals per surface area $\left(10 \mathrm{~cm}^{2}\right)$.

\subsection{Statistical analysis}

Biological assemblage descriptors (total abundance and species richness) were estimated for each sample. Differences in species richness and abundance patterns during months of the study period (the fixed factor) were tested through a oneway ANOVA, after verifying normality using the KolmogorovSmirnov test and Levene's test for homogeneity of variances. When the requirements of normality and/or homogeneity of variances were not fulfilled, the Kruskal-Wallis ANOVA (KW ANOVA) test was used instead.

To visualize affinities in assemblage structure through the study period, n-MDS (non-metric multidimensional scaling) was carried out on square root transformed abundance data via the Bray-Curtis similarity index. The ANOSIM routine (Clarke, 1993) was used to explore the significance of differences among months.

The relationship between environmental variables and meiofaunal community structure was explored using BIOENV procedures. All multivariate analyses were carried out by means of the PRIMER 5.2. package (Clarke and Gorley, 2001).

\section{Results}

\subsection{Sedimentary variables}

The sampling site was dominated by medium sands $(0.25-$ $0.5 \mathrm{~mm})$, ranging from a minimum dominance of $31.7 \%(\mathrm{Au}-$ gust 2000) to a maximum of $64.4 \%$ (December 2000). Fine sand$\mathrm{s}$ were the second sedimentary fraction in importance, ranging from $16.6 \%$ (May 2000) to 55.9\% (September 2000). The remaining sediment types were scarce. Organic matter content was characterized by a mean percentage of $0.90 \%$ throughout the study period, with a maximum content of $1.68 \%$ (May 2000) and a minimum content of $0.50 \%$ (October and November 2000). Nitrogen concentration obtained a mean value of $0.009 \%$, ranging from $0.010 \%$ (June 2000, September 2000, and April 2001) to 0.008\% (October 2000-February 2001) (Table 1).

\subsection{Meiofauna}

A total of 15 taxonomic groups were collected: nematodes, oligochaetes, polychaetes, copepods, turbellarians, tanaids, amphipods, cnidarians, echinoids, misids, ostracods, acari, ne-

Table 1. Values of sedimentary variables throughout the study period in the Los Abrigos subtidal

\begin{tabular}{lcccccccccccccc}
\hline & May & June & July & August & September & October & November & December & January & February & March & April \\
& 2000 & 2000 & 2000 & 2000 & 2000 & 2000 & 2000 & 2000 & 2001 & 2001 & 2001 & 2001 \\
\hline Nitrogen/\% & 0.009 & 0.01 & 0.009 & 0.009 & 0.01 & 0.008 & 0.008 & 0.008 & 0.008 & 0.008 & 0.009 & 0.01 \\
Carbonates/\% & 3.08 & 6.84 & 5.13 & 9.57 & 7.86 & 4.62 & 1.54 & 5.47 & 7.01 & 5.98 & 6.32 & 7.18 \\
Organic matter/\% & 1.68 & 1.54 & 1.31 & 0.77 & 0.74 & 0.51 & 0.5 & 0.78 & 0.7 & 0.54 & 0.91 & 0.85 \\
Gravels/\% & 9.82 & 5.42 & 5.64 & 12.24 & 2.56 & 0.46 & 2.28 & 0.48 & 1.56 & 1.56 & 7.83 & 5.72 \\
Very coarse sands/\% & 6.98 & 2.44 & 1.72 & 4.87 & 1.18 & 0.92 & 1.35 & 1.09 & 0.65 & 1.55 & 3.72 & 1.58 \\
Coarse sands/\% & 7.71 & 3.62 & 5.29 & 5.49 & 1.17 & 1.61 & 2.11 & 2.58 & 1.06 & 2.48 & 3.4 & 1.25 \\
Medium sands/\% & 58.5 & 52.04 & 57.41 & 31.7 & 35.61 & 54.21 & 50.61 & 64.38 & 50.82 & 52.04 & 42.63 & 34.44 \\
Fine sands/\% & 16.57 & 32.36 & 25.84 & 41.91 & 55.89 & 40.76 & 41.89 & 30.32 & 44.5 & 40.32 & 40.41 & 54.08 \\
Very fine sands/\% & 0.39 & 3.88 & 3.81 & 3.46 & 3.21 & 1.94 & 1.63 & 1.07 & 1.33 & 1.88 & 1.87 & 2.75 \\
Silt/clay/\% & 0.03 & 0.25 & 0.29 & 0.32 & 0.38 & 0.11 & 0.13 & 0.07 & 0.09 & 0.17 & 0.14 & 0.17 \\
\hline
\end{tabular}


merteans, decapods, and kinorhynchs. Nematodes were the most abundant group with 38504 individuals $(91.11 \%$ of the overall abundance), ranging from $78 \%$ in May 2000 to $97.34 \%$ in February 2001. The second and third most abundant taxonom- ic groups were oligochaetes and polychaetes, with 683 (1.66\%) and $679(1.65 \%)$ individuals, respectively. The remaining taxonomical groups (copepods, turbellarians, ostracods, acari, nemerteans, decapods, and kinorhynchs) were scarce (Table 2).

Table 2. Mean abundances ( \pm SE) of meiofaunal species in the Los Abrigos subtidal throughout the study period

\begin{tabular}{|c|c|c|c|c|c|c|c|}
\hline Group & Species & $\begin{array}{l}\text { May } \\
2000\end{array}$ & $\begin{array}{l}\text { June } \\
2000\end{array}$ & $\begin{array}{l}\text { July } \\
2000\end{array}$ & $\begin{array}{c}\text { August } \\
2000\end{array}$ & $\begin{array}{c}\text { September } \\
2000\end{array}$ & $\begin{array}{c}\text { October } \\
2000\end{array}$ \\
\hline Nematoda & Actarjania sp. & 0 & 0 & 0 & 0 & 0 & 0 \\
\hline Nematoda & Actarjania sp. 2 & 0 & 0 & 0 & 0 & 0 & 0 \\
\hline Nematoda & Ammotheristus sp. & $0.2 \pm 0.4$ & 0 & 0 & 0 & 0 & 0 \\
\hline Nematoda & Anticomasp. & 0 & 0 & $1.4 \pm 2.2$ & $0.4 \pm 0.9$ & 0 & 0 \\
\hline Nematoda & Ascolaimus sp. & 0 & $0.2 \pm 0.4$ & 0 & 0 & 0 & 0 \\
\hline Nematoda & Calyptronema sp. & $1.8 \pm 2.5$ & 0 & 0 & 0 & 0 & 0 \\
\hline Nematoda & Catanema sp. & $41.2 \pm 80.1$ & $179.8 \pm 55.6$ & $58.6 \pm 52.1$ & $45 \pm 11.2$ & $97.2 \pm 49.1$ & $161 \pm 70$ \\
\hline Nematoda & Ceramonema aff. yunfengi & 0 & $0.2 \pm 0.4$ & $0.2 \pm 0.4$ & 0 & $0.8 \pm 1.8$ & $3.8 \pm 5.5$ \\
\hline Nematoda & Metadasynemella sp. & $0.8 \pm 1.8$ & $4 \pm 7.8$ & 0 & $1.2 \pm 1.1$ & $1 \pm 2.2$ & $0.8 \pm 1.8$ \\
\hline Nematoda & Choniolaimus sp. & $0.8 \pm 1.8$ & $1.2 \pm 2.7$ & 0 & 0 & 0 & 0 \\
\hline Nematoda & Chromadorita sp. & $0.4 \pm 0.9$ & 0 & 0 & 0 & 0 & 0 \\
\hline Nematoda & Cobbia sp. & 0 & $0.4 \pm 0.5$ & 0 & 0 & $0.8 \pm 1.8$ & $1.4 \pm 1.9$ \\
\hline Nematoda & Cobbia aff. truncata & 0 & 0 & 0 & 0 & 0 & 0 \\
\hline Nematoda & Daptonema aff. fallax & 0 & 0 & 0 & 0 & 0 & 0 \\
\hline Nematoda & Daptonema aff. hirsutum & $4.2 \pm 3.3$ & $12.8 \pm 8.6$ & $0.4 \pm 0.9$ & $18.8 \pm 21.1$ & $14.6 \pm 19.4$ & $17 \pm 5.9$ \\
\hline Nematoda & Dasynemoides sp. & $1.6 \pm 1.1$ & $6.6 \pm 2.6$ & 0 & $1 \pm 1.4$ & $3 \pm 3.1$ & $2.2 \pm 3.5$ \\
\hline Nematoda & Desmodorella aff. tenuispiculum & $0.6 \pm 0.9$ & $0.6 \pm 1.3$ & 0 & 0 & 0 & 0 \\
\hline Nematoda & Diodontolaimus aff. sabulosus & 0 & 0 & 0 & 0 & 0 & $0.8 \pm 1.8$ \\
\hline Nematoda & Enoploides gryphus & $2.6 \pm 2.9$ & $1.6 \pm 0.9$ & 0 & $2.6 \pm 2.4$ & $2.8 \pm 3.8$ & $2 \pm 3.1$ \\
\hline Nematoda & Enoplolaimus aff. propinquus & $4.2 \pm 8.3$ & 0 & 0 & $1.2 \pm 1.1$ & 0 & 0 \\
\hline Nematoda & Enoplus sp. 2 & $0.4 \pm 0.9$ & 0 & 0 & 0 & 0 & 0 \\
\hline Nematoda & Gerlachius sp. & 0 & 0 & 0 & 0 & 0 & 0 \\
\hline Nematoda & Gonionchus aff. cumbraensis & $0.8 \pm 1.1$ & $0.2 \pm 0.4$ & 0 & $0.4 \pm 0.9$ & $2 \pm 1.9$ & $6 \pm 9.7$ \\
\hline Nematoda & Halalaimus sp. 3 & 0 & 0 & 0 & 0 & 0 & 0 \\
\hline Nematoda & Kraspedonemasp. & 0 & 0 & 0 & 0 & 0 & 0 \\
\hline Group & Species & $\begin{array}{c}\text { November } \\
2000 \\
\end{array}$ & $\begin{array}{c}\text { December } \\
2000 \\
\end{array}$ & $\begin{array}{c}\text { January } \\
2001 \\
\end{array}$ & $\begin{array}{c}\text { February } \\
2001 \\
\end{array}$ & $\begin{array}{c}\text { March } \\
2001 \\
\end{array}$ & $\begin{array}{l}\text { April } \\
2001 \\
\end{array}$ \\
\hline Nematoda & Actarjania sp. & $1.4 \pm 3.1$ & 0 & 0 & 0 & 0 & 0 \\
\hline Nematoda & Actarjaniasp. 2 & 0 & $1.4 \pm 1.9$ & 0 & 0 & $0.8 \pm 1.8$ & 0 \\
\hline Nematoda & Ammotheristus sp. & 0 & 0 & 0 & 0 & 0 & 0 \\
\hline Nematoda & Anticomasp. & 0 & 0 & 0 & 0 & $1.4 \pm 3.1$ & $0.8 \pm 1.8$ \\
\hline Nematoda & Ascolaimus sp. & 0 & 0 & 0 & 0 & 0 & 0 \\
\hline Nematoda & Calyptronema sp. & $0.6 \pm 1.3$ & $0.8 \pm 1.8$ & 0 & 0 & 0 & 0 \\
\hline Nematoda & Catanema sp. & $221.2 \pm 168.6$ & $103.2 \pm 64.2$ & $165.8 \pm 89.4$ & $333.8 \pm 94$ & $196.6 \pm 99$ & $177.8 \pm 68.8$ \\
\hline Nematoda & Ceramonema aff. yunfengi & $1.8 \pm 2.5$ & $4.6 \pm 4$ & 0 & $2 \pm 3.1$ & 0 & 0 \\
\hline Nematoda & Metadasynemella sp. & 0 & $2.4 \pm 3.3$ & 0 & $4.8 \pm 5.8$ & $1.6 \pm 2.2$ & 0 \\
\hline Nematoda & Choniolaimus sp. & 0 & 0 & 0 & 0 & $1.6 \pm 3.6$ & 0 \\
\hline Nematoda & Chromadorita sp. & $0.6 \pm 1.3$ & $0.8 \pm 1.8$ & 0 & 0 & 0 & 0 \\
\hline Nematoda & Cobbia sp. & $1 \pm 2.2$ & 0 & 0 & 0 & 0 & 0 \\
\hline Nematoda & Cobbia aff. truncata & 0 & $1.6 \pm 2.2$ & 0 & $0.6 \pm 1.3$ & $0.8 \pm 1.8$ & 0 \\
\hline Nematoda & Daptonema aff. fallax & $0.8 \pm 1.8$ & 0 & 0 & 0 & 0 & 0 \\
\hline Nematoda & Daptonema aff. hirsutum & $40.6 \pm 49.6$ & $54 \pm 25.2$ & $74.6 \pm 50.2$ & $26.6 \pm 16$ & $58 \pm 36$ & $16.8 \pm 10.2$ \\
\hline Nematoda & Dasynemoides sp. & $1 \pm 2.2$ & $4 \pm 2.5$ & 0 & $3.4 \pm 4.1$ & $0.8 \pm 1.8$ & $0.8 \pm 1.8$ \\
\hline Nematoda & Desmodorella aff. tenuispiculum & 0 & 0 & 0 & 0 & 0 & 0 \\
\hline Nematoda & Diodontolaimus aff. sabulosus & 0 & 0 & 0 & 0 & 0 & 0 \\
\hline Nematoda & Enoploides gryphus & $6 \pm 10.8$ & $28.2 \pm 12$ & $7.6 \pm 6.7$ & $9.8 \pm 10.5$ & $5.4 \pm 4.2$ & $1.8 \pm 2.5$ \\
\hline Nematoda & Enoplolaimus aff. propinquus & 0 & 0 & 0 & 0 & 0 & 0 \\
\hline Nematoda & Enoplus sp. 2 & 0 & 0 & 0 & 0 & 0 & 0 \\
\hline Nematoda & Gerlachius sp. & $1 \pm 2.2$ & 0 & 0 & 0 & 0 & 0 \\
\hline Nematoda & Gonionchus aff. cumbraensis & $2.2 \pm 2.2$ & $2 \pm 3.1$ & $0.8 \pm 1.8$ & 0 & $2.2 \pm 3.2$ & $1.6 \pm 3.6$ \\
\hline Nematoda & Halalaimus sp. & 0 & 0 & 0 & 0 & 0 & $1.6 \pm 3.6$ \\
\hline Nematoda & Kraspedonema sp. & 0 & 0 & 0 & 0 & $0.8 \pm 1.8$ & 0 \\
\hline Nematoda & Laimella aff. longicaudata & 0 & 0 & 0 & 0 & 0 & $1.6 \pm 3.6$ \\
\hline
\end{tabular}


Continued from Table 2

\begin{tabular}{|c|c|c|c|c|c|c|c|}
\hline Group & Species & $\begin{array}{l}\text { May } \\
2000\end{array}$ & $\begin{array}{l}\text { June } \\
2000\end{array}$ & $\begin{array}{l}\text { July } \\
2000\end{array}$ & $\begin{array}{c}\text { August } \\
2000\end{array}$ & $\begin{array}{c}\text { September } \\
2000\end{array}$ & $\begin{array}{l}\text { October } \\
2000\end{array}$ \\
\hline Nematoda & Mesacanthion diplechma & $1.8 \pm 2.5$ & $0.6 \pm 0.5$ & 0 & 0 & $1 \pm 2.2$ & $2.2 \pm 3.2$ \\
\hline Nematoda & Metachromadorasp. & 0 & 0 & 0 & $0.4 \pm 0.9$ & 0 & 0 \\
\hline Nematoda & Microlaimus sp. 1 & $0.6 \pm 0.9$ & 0 & 0 & 0 & 0 & 0 \\
\hline Nematoda & Monoposthia aff. mirabilis & $4.4 \pm 5.9$ & 0 & 0 & $2.2 \pm 3.9$ & $2.8 \pm 4.8$ & 0 \\
\hline Nematoda & Monoposthiasp. & $0.4 \pm 0.9$ & $0.2 \pm 0.4$ & 0 & 0 & 0 & 0 \\
\hline Nematoda & Odontophora aff. longisetosa & $0.4 \pm 0.9$ & 0 & 0 & $0.4 \pm 0.9$ & 0 & $0.6 \pm 1.3$ \\
\hline Nematoda & Odontophora aff. villoti & 0 & 0 & 0 & $5.4 \pm 12.1$ & $0.6 \pm 1.3$ & 0 \\
\hline Nematoda & Odontophora aff. wieseri & $3.2 \pm 4.1$ & $4 \pm 2$ & 0 & $0.4 \pm 0.9$ & $4.8 \pm 5$ & $5.4 \pm 4.9$ \\
\hline Nematoda & Oncholaimellus calvadosicus & $1.4 \pm 2.6$ & $1.6 \pm 2.3$ & $2 \pm 4.5$ & $27.8 \pm 35.3$ & $20 \pm 15.8$ & $22.4 \pm 9.8$ \\
\hline Nematoda & Oncholaimus campylocercoides & $7.4 \pm 8.6$ & $10.6 \pm 2.7$ & $1 \pm 2.2$ & $35 \pm 56.5$ & $20.4 \pm 10.9$ & $66.6 \pm 27.8$ \\
\hline Nematoda & Onyx aff. perfectus & $1.2 \pm 1.8$ & 0 & 0 & 0 & 0 & 0 \\
\hline Nematoda & Paracomesomasp. & $0.4 \pm 0.9$ & 0 & 0 & 0 & 0 & 0 \\
\hline Nematoda & Paralinhomoeus aff. tenuicaudatum & $0.2 \pm 0.4$ & $0.8 \pm 1.8$ & 0 & 0 & $0.6 \pm 1.3$ & $1.4 \pm 1.9$ \\
\hline Nematoda & Paralinhomoeus aff. uniovarium & 0 & 0 & 0 & 0 & 0 & 0 \\
\hline Nematoda & Paralinhomoeussp. & $1 \pm 1.7$ & $4.8 \pm 8.1$ & $5.8 \pm 6.6$ & $8.8 \pm 5.4$ & $8.8 \pm 7.7$ & $14 \pm 12.3$ \\
\hline Nematoda & Paramesonchium sp. & $2.4 \pm 5.4$ & $10.2 \pm 3.7$ & $1.6 \pm 1.5$ & $23 \pm 22.8$ & $21.6 \pm 20.3$ & $49.8 \pm 40$ \\
\hline Nematoda & Paramonohystera aff. riemanni & 0 & 0 & 0 & 0 & 0 & $0.6 \pm 1.3$ \\
\hline Nematoda & Pareurystomina aff. bissonettei & 0 & $0.2 \pm 0.4$ & $0.6 \pm 1.3$ & 0 & 0 & $1.4 \pm 1.9$ \\
\hline Nematoda & Phanodermopsissp. & $0.4 \pm 0.9$ & 0 & 0 & 0 & 0 & 0 \\
\hline Nematoda & Marylynnia sp. & 0 & 0 & 0 & 0 & 0 & 0 \\
\hline Nematoda & Pomponema aff. compactum & $3.8 \pm 5$ & $2 \pm 2.1$ & $0.8 \pm 1.1$ & $11.4 \pm 19.7$ & $6.2 \pm 5.7$ & $6.8 \pm 10$ \\
\hline Nematoda & Pomponema aff. reducta & 0 & $16.2 \pm 6.2$ & $5.4 \pm 6.5$ & $2.8 \pm 2.6$ & $6 \pm 3.4$ & $12.4 \pm 8.7$ \\
\hline Nematoda & Promonhysterasp. & 0 & 0 & 0 & 0 & 0 & $0.6 \pm 1.3$ \\
\hline Nematoda & Pseudochromadorasp. 2 & $0.4 \pm 0.9$ & $0.2 \pm 0.4$ & $0.8 \pm 1.1$ & $7.8 \pm 7.5$ & $19.8 \pm 14.3$ & $7.4 \pm 4.2$ \\
\hline Nematoda & Rhabdodemania aff. imer & $0.2 \pm 0.4$ & 0 & $0.6 \pm 0.9$ & 0 & 0 & 0 \\
\hline Nematoda & Rhabdodemania sp2 & 0 & $0.2 \pm 0.4$ & 0 & 0 & 0 & 0 \\
\hline Nematoda & Sabatieria aff. longisetosa & $1.4 \pm 2.6$ & 0 & 0 & 0 & 0 & 0 \\
\hline Group & Species & $\begin{array}{c}\text { November } \\
2000\end{array}$ & $\begin{array}{c}\text { December } \\
2000\end{array}$ & $\begin{array}{c}\text { January } \\
2001\end{array}$ & $\begin{array}{c}\text { February } \\
2001\end{array}$ & $\begin{array}{c}\text { March } \\
2001\end{array}$ & $\begin{array}{l}\text { April } \\
2001\end{array}$ \\
\hline Nematoda & Laimella aff. longicaudata & 0 & 0 & 0 & 0 & 0 & 0 \\
\hline Nematoda & Mesacanthion diplechma & $1.8 \pm 4$ & $6 \pm 2.5$ & $1 \pm 2.2$ & 0 & $0.8 \pm 1.8$ & $1 \pm 2.2$ \\
\hline Nematoda & Metachromadora sp. & 0 & 0 & 0 & 0 & 0 & 0 \\
\hline Nematoda & Microlaimus sp. 1 & 0 & 0 & 0 & 0 & 0 & 0 \\
\hline Nematoda & Monoposthia aff. mirabilis & $1.2 \pm 2.7$ & $3.6 \pm 5.1$ & $2.6 \pm 4$ & $4.6 \pm 8.2$ & 0 & 0 \\
\hline Nematoda & Monoposthiasp. & 0 & $0.8 \pm 1.8$ & 0 & $1.2 \pm 2.7$ & 0 & 0 \\
\hline Nematoda & Odontophora aff. longisetosa & $5.6 \pm 3.7$ & $16.4 \pm 13.1$ & $11.8 \pm 5.3$ & $6.8 \pm 3.9$ & $3.2 \pm 5.2$ & 0 \\
\hline Nematoda & Odontophora aff. villoti & 0 & 0 & $5 \pm 11.2$ & 0 & 0 & $0.8 \pm 1.8$ \\
\hline Nematoda & Odontophora aff. wieseri & $1.4 \pm 3.1$ & 0 & 0 & 0 & 0 & 0 \\
\hline Nematoda & Oncholaimellus calvadosicus & $28.2 \pm 45.6$ & $54 \pm 17$ & $14.6 \pm 15.4$ & $10.2 \pm 6.6$ & $14.6 \pm 6.3$ & $6 \pm 6.7$ \\
\hline Nematoda & Oncholaimus campylocercoides & $126.4 \pm 84.4$ & $184.8 \pm 101.6$ & $44.2 \pm 44.1$ & $19 \pm 12.4$ & $27.2 \pm 12.6$ & $9.2 \pm 7.2$ \\
\hline Nematoda & Onyx aff. perfectus & 0 & $0.8 \pm 1.8$ & 0 & 0 & 0 & 0 \\
\hline Nematoda & Paracomesomasp. & 0 & $14.4 \pm 11.8$ & $10.4 \pm 7.9$ & $8 \pm 7.6$ & $1.6 \pm 3.6$ & 0 \\
\hline Nematoda & Paralinhomoeus aff. tenuicaudatum & $3.8 \pm 6.1$ & $3.6 \pm 8$ & 0 & 0 & 0 & 0 \\
\hline Nematoda & Paralinhomoeus aff. uniovarium & $1 \pm 2.2$ & 0 & 0 & 0 & $0.8 \pm 1.8$ & 0 \\
\hline Nematoda & Paralinhomoeus sp. & $3.8 \pm 3.9$ & $6.4 \pm 5.1$ & $8 \pm 11.2$ & $2.8 \pm 3.8$ & $11.2 \pm 5.5$ & $23.6 \pm 25.2$ \\
\hline Nematoda & Paramesonchium sp. & $33.2 \pm 25.2$ & $35.6 \pm 19.4$ & $37 \pm 32.9$ & $11.8 \pm 12$ & $11.4 \pm 7$ & $5.8 \pm 2.6$ \\
\hline Nematoda & Paramonohystera aff. riemanni & 0 & $11.8 \pm 8.3$ & $1.8 \pm 4$ & $1.8 \pm 2.7$ & $1.6 \pm 2.2$ & $1 \pm 2.2$ \\
\hline Nematoda & Pareurystomina aff. bissonettei & $1 \pm 2.2$ & $0.8 \pm 1.8$ & $1.6 \pm 2.2$ & $1.8 \pm 2.7$ & $1.6 \pm 2.2$ & 0 \\
\hline Nematoda & Phanodermopsis sp. & 0 & 0 & 0 & 0 & 0 & 0 \\
\hline Nematoda & Marylynniasp. & $1 \pm 2.2$ & 0 & 0 & 0 & 0 & $1 \pm 2.2$ \\
\hline Nematoda & Pomponema aff. compactum & $4.4 \pm 3.6$ & $38.4 \pm 31.5$ & $31.6 \pm 54.1$ & $5.6 \pm 10.9$ & $3.8 \pm 5.3$ & $3.2 \pm 3.7$ \\
\hline Nematoda & Pomponema aff. reducta & $12 \pm 8.9$ & $3.8 \pm 4.5$ & $37.4 \pm 17.1$ & $22.2 \pm 22$ & $14.6 \pm 8.1$ & $13.8 \pm 9.9$ \\
\hline Nematoda & Promonhysterasp. & 0 & 0 & 0 & 0 & 0 & 0 \\
\hline Nematoda & Pseudochromadorasp. 2 & $5.6 \pm 6.9$ & $7.2 \pm 7.2$ & $36.8 \pm 27.6$ & $7 \pm 8$ & $15 \pm 18.1$ & $9 \pm 17.5$ \\
\hline Nematoda & Rhabdodemania aff. imer & 0 & 0 & 0 & 0 & 0 & 0 \\
\hline Nematoda & Rhabdodemania sp2 & 0 & 0 & 0 & 0 & 0 & 0 \\
\hline Nematoda & Sabatieria aff. longisetosa & 0 & 0 & 0 & 0 & 0 & 0 \\
\hline Nematoda & Sabatieria aff. elongata & $11.8 \pm 18.4$ & $59.4 \pm 13.2$ & $5.6 \pm 4.6$ & $33.6 \pm 15.3$ & $63.8 \pm 30.6$ & $151 \pm 41$ \\
\hline
\end{tabular}


Continued from Table 2

\begin{tabular}{|c|c|c|c|c|c|c|c|}
\hline Group & Species & $\begin{array}{l}\text { May } \\
2000\end{array}$ & $\begin{array}{l}\text { June } \\
2000\end{array}$ & $\begin{array}{l}\text { July } \\
2000\end{array}$ & $\begin{array}{c}\text { August } \\
2000\end{array}$ & $\begin{array}{c}\text { September } \\
2000\end{array}$ & $\begin{array}{c}\text { October } \\
2000\end{array}$ \\
\hline Nematoda & Sabatieria sp. 3 & 0 & 0 & 0 & 0 & 0 & 0 \\
\hline Nematoda & Setosabatieria aff. hilarula & 0 & 0 & 0 & 0 & 0 & 0 \\
\hline Nematoda & Siphonolaimus aff. niger & 0 & 0 & 0 & 0 & 0 & 0 \\
\hline Nematoda & Siphonolaimus sp. 1 & $1.6 \pm 3.6$ & $2.2 \pm 2.7$ & $85.2 \pm 41.9$ & $77.2 \pm 56.2$ & $104 \pm 22.4$ & $50.2 \pm 38.9$ \\
\hline Nematoda & Siphonolaimus sp. 2 & $3.4 \pm 6.5$ & $42 \pm 12.1$ & $447 \pm 195.5$ & $237.6 \pm 156.3$ & $280.8 \pm 114.4$ & $82.2 \pm 50.7$ \\
\hline Nematoda & Southerniasp. & 0 & 0 & 0 & 0 & 0 & $0.6 \pm 1.3$ \\
\hline Nematoda & Spirinia parasitifera & $0.2 \pm 0.4$ & 0 & 0 & 0 & 0 & 0 \\
\hline Nematoda & Synonchiella aff. forceps & $6.8 \pm 6.7$ & $21.8 \pm 3.9$ & $3.4 \pm 4.5$ & $5.6 \pm 4.2$ & $7.6 \pm 5.1$ & $22.2 \pm 12.4$ \\
\hline Nematoda & Tarvaia sp. & 0 & $0.4 \pm 0.5$ & 0 & 0 & 0 & 0 \\
\hline Nematoda & Thalassironus sp. 1 & 0 & 0 & $1 \pm 2.2$ & $0.6 \pm 1.3$ & $0.6 \pm 1.3$ & $0.8 \pm 1.8$ \\
\hline Nematoda & Thalassironus sp. 2 & 0 & 0 & 0 & 0 & 0 & $0.8 \pm 1.8$ \\
\hline Nematoda & Theristussp. 2 & 0 & 0 & 0 & 0 & 0 & $0.6 \pm 1.3$ \\
\hline Nematoda & Theristus sp. 3 & 0 & 0 & 0 & 0 & 0 & 0 \\
\hline Nematoda & Trefusia sp. & 0 & 0 & 0 & 0 & 0 & 0 \\
\hline Nematoda & Trileptium aff. parisetum & $1.8 \pm 1.6$ & $1 \pm 2.2$ & $0.6 \pm 0.9$ & 0 & 0 & 0 \\
\hline Nematoda & Vasostoma sp. & 0 & 0 & $0.2 \pm 0.4$ & $3.6 \pm 8$ & 0 & $1.2 \pm 1.6$ \\
\hline Nematoda & Viscosia glabra & 0 & 0 & 0 & $0.4 \pm 0.9$ & 0 & 0 \\
\hline Nematoda & Xyala striata & $1.4 \pm 2.2$ & $0.6 \pm 1.3$ & 0 & 0 & 0 & $0.6 \pm 1.3$ \\
\hline Copepoda & Tryphonemasp. & $2.8 \pm 3$ & $0.2 \pm 0.4$ & 0 & $0.6 \pm 0.9$ & $0.2 \pm 0.4$ & 0 \\
\hline Copepoda & Halectinosoma sp. & $1.2 \pm 1.6$ & $1.2 \pm 1.3$ & 0 & $2.2 \pm 3.3$ & $3.2 \pm 5.5$ & $2.6 \pm 3.2$ \\
\hline Copepoda & Ectinosomatidaesp. & $7.2 \pm 10.4$ & $0.2 \pm 0.4$ & 0 & $0.6 \pm 1.3$ & 0 & $0.8 \pm 1.8$ \\
\hline Copepoda & Tisbe aff. bulbisetosa & $0.4 \pm 0.9$ & 0 & 0 & 0 & 0 & 0 \\
\hline Copepoda & Intermedopsyllussp. & $0.6 \pm 0.9$ & 0 & 0 & 0 & 0 & 0 \\
\hline Copepoda & Asellopsis sp. & $0.4 \pm 0.9$ & 0 & 0 & 0 & 0 & 0 \\
\hline Copepoda & Harpacticus aff. flexus & $0.8 \pm 1.8$ & 0 & 0 & $1 \pm 2.2$ & $2 \pm 2.5$ & $3 \pm 2.5$ \\
\hline Copepoda & Canuella aff. perplexa & 0 & $0.6 \pm 1.3$ & $0.6 \pm 1.3$ & $1.2 \pm 2.2$ & $0.8 \pm 1.1$ & $1 \pm 1.4$ \\
\hline Copepoda & Paralaophontesp. & 0 & 0 & 0 & 0 & $0.4 \pm 0.5$ & 0 \\
\hline \multirow[t]{2}{*}{ Group } & Species & November & December & January & February & March & April \\
\hline & & 2000 & 2000 & 2001 & 2001 & 2001 & 2001 \\
\hline Nematoda & Sabatieria aff. elongata & $93.2 \pm 31.1$ & $90.6 \pm 37.7$ & $49 \pm 23$ & $49.8 \pm 42.6$ & $91.4 \pm 49.1$ & $35.6 \pm 32.8$ \\
\hline Nematoda & Sabatieria sp. & 0 & $1.4 \pm 3.1$ & 0 & 0 & 0 & 0 \\
\hline Nematoda & Setosabatieria aff. hilarula & 0 & $0.8 \pm 1.8$ & 0 & 0 & 0 & 0 \\
\hline Nematoda & Siphonolaimus aff. niger & 0 & 0 & $1 \pm 2.2$ & 0 & 0 & 0 \\
\hline Nematoda & Siphonolaimus sp. 1 & $61.8 \pm 91.4$ & $16.6 \pm 19.2$ & $52.8 \pm 42.5$ & $52.6 \pm 51$ & $63.2 \pm 30.4$ & $73.2 \pm 20.4$ \\
\hline Nematoda & Siphonolaimus sp. 2 & $114.6 \pm 138.2$ & $15.8 \pm 18.2$ & $192.6 \pm 193$ & $159.6 \pm 130.8$ & $179.4 \pm 86.2$ & $369.6 \pm 173.2$ \\
\hline Nematoda & Southerniasp. & 0 & 0 & 0 & 0 & 0 & 0 \\
\hline Nematoda & Spirinia parasitifera & 0 & $6 \pm 2.5$ & $5 \pm 5.8$ & $1.2 \pm 2.7$ & 0 & 0 \\
\hline Nematoda & Synonchiella aff. forceps & $11.2 \pm 12.1$ & $19.2 \pm 5.1$ & $11.8 \pm 8$ & $20 \pm 12.4$ & $23.6 \pm 12.6$ & $12.4 \pm 16.3$ \\
\hline Nematoda & Tarvaia sp. & 0 & 0 & 0 & 0 & 0 & 0 \\
\hline Nematoda & Thalassironus sp. 1 & 0 & 0 & 0 & 0 & 0 & 0 \\
\hline Nematoda & Thalassironus sp. 2 & $0.8 \pm 1.8$ & 0 & 0 & 0 & 0 & $0.8 \pm 1.8$ \\
\hline Nematoda & Theristus sp. 2 & 0 & 0 & 0 & 0 & 0 & 0 \\
\hline Nematoda & Theristus sp. 3 & 0 & $1.6 \pm 3.6$ & 0 & 0 & 0 & 0 \\
\hline Nematoda & Trefusia sp. & 0 & $2.2 \pm 4.9$ & 0 & 0 & 0 & 0 \\
\hline Nematoda & Trileptium aff. parisetum & 0 & $0.8 \pm 1.8$ & 0 & 0 & 0 & $0.8 \pm 1.8$ \\
\hline Nematoda & Vasostoma sp. & $1.6 \pm 2.3$ & 0 & $0.8 \pm 1.8$ & $0.8 \pm 1.8$ & 0 & 0 \\
\hline Nematoda & Viscosia glabra & 0 & 0 & 0 & 0 & $2.4 \pm 3.6$ & $1 \pm 2.2$ \\
\hline Nematoda & Xyala striata & $1.6 \pm 2.3$ & $7.4 \pm 8.5$ & 0 & $1.2 \pm 2.7$ & $0.8 \pm 1.8$ & $1 \pm 2.2$ \\
\hline Copepoda & Tryphonemasp. & $0.4 \pm 0.5$ & $1 \pm 1$ & $0.4 \pm 0.5$ & $0.2 \pm 0.4$ & $0.6 \pm 0.9$ & 0 \\
\hline Copepoda & Halectinosoma sp. & $4.4 \pm 4.6$ & $9.2 \pm 4.7$ & $7.2 \pm 3.3$ & $3.4 \pm 2.3$ & $9.4 \pm 2.5$ & $7 \pm 10.3$ \\
\hline Copepoda & Ectinosomatidaesp. & 0 & $0.8 \pm 1.3$ & 0 & 0 & 0 & 0 \\
\hline Copepoda & Tisbe aff. bulbisetosa & 0 & 0 & 0 & 0 & 0 & 0 \\
\hline Copepoda & Intermedopsyllus sp. & 0 & 0 & 0 & 0 & 0 & 0 \\
\hline Copepoda & Asellopsis sp. & 0 & 0 & 0 & 0 & 0 & \\
\hline Copepoda & Harpacticus aff. flexus & $0.2 \pm 0.4$ & $24 \pm 12.3$ & $1.4 \pm 3.1$ & 0 & 0 & $0.2 \pm 0.4$ \\
\hline Copepoda & Canuella aff. perplexa & 0 & 0 & 0 & $1 \pm 1$ & $8.4 \pm 4.9$ & $7.2 \pm 12.8$ \\
\hline Copepoda & Paralaophontesp. & 0 & 0 & 0 & 0 & 0 & 0 \\
\hline Polychaeta & Aricidea (Acmira) catherinae & 0 & 0 & 0 & 0 & 0 & 0 \\
\hline
\end{tabular}


Continued from Table 2

\begin{tabular}{|c|c|c|c|c|c|c|c|}
\hline Group & Species & $\begin{array}{l}\text { May } \\
2000\end{array}$ & $\begin{array}{l}\text { June } \\
2000\end{array}$ & $\begin{array}{l}\text { July } \\
2000\end{array}$ & $\begin{array}{c}\text { August } \\
2000\end{array}$ & $\begin{array}{c}\text { September } \\
2000\end{array}$ & $\begin{array}{c}\text { October } \\
2000\end{array}$ \\
\hline Polychaeta & Brania arminii & 0 & $0.4 \pm 0.9$ & 0 & 0 & 0 & 0 \\
\hline Polychaeta & Capitomastus minimus & 0 & $0.4 \pm 0.9$ & 0 & $0.8 \pm 1.3$ & $0.2 \pm 0.4$ & 0 \\
\hline Polychaeta & Notomastus latericeus & $0.2 \pm 0.4$ & 0 & 0 & 0 & $0.2 \pm 0.4$ & 0 \\
\hline Polychaeta & Cauleriella bioculata & 0 & 0 & $0.2 \pm 0.4$ & 0 & 0 & 0 \\
\hline Polychaeta & Cirriformia tentaculata & 0 & 0 & 0 & 0 & 0 & 0 \\
\hline Polychaeta & Cirrophorus furcatus & 0 & 0 & 0 & 0 & 0 & $0.4 \pm 0.9$ \\
\hline Polychaeta & Cirrophorus perdidoensis & $0.2 \pm 0.4$ & 0 & 0 & 0 & 0 & 0 \\
\hline Polychaeta & Dispio uncinata & 0 & $0.4 \pm 0.9$ & $0.4 \pm 0.9$ & 0 & 0 & 0 \\
\hline Polychaeta & Exogone breviantennata & 0 & 0 & 0 & 0 & 0 & $0.2 \pm 0.4$ \\
\hline Polychaeta & Microphthalmus pseudoaberrans & $0.4 \pm 0.9$ & 0 & 0 & 0 & 0.4 & 0.8 \\
\hline Polychaeta & Ophelia bicornis & $0.2 \pm 0.4$ & 0 & 0 & 0 & 0 & 0 \\
\hline Polychaeta & Protodrilus aff. rubropharyngeus & $0.2 \pm 0.4$ & 0 & 0 & 0 & 0 & 0 \\
\hline Polychaeta & Parapionosyllis macaronesiensis & 0 & 0 & 0 & 0 & 0 & 0 \\
\hline Polychaeta & Parapionosyllis minuta & 0 & $0.2 \pm 0.4$ & 0 & 0 & 0 & 0 \\
\hline Polychaeta & Parapionosyllis n. sp. & 0 & 0 & $0.2 \pm 0.4$ & $0.2 \pm 0.4$ & 0 & 0 \\
\hline Polychaeta & Perinereis cultrifera & 0 & 0 & 0 & 0 & 0 & 0 \\
\hline Polychaeta & Periquesta canariensis & $0.4 \pm 0.5$ & $9.6 \pm 20.4$ & $0.2 \pm 0.4$ & $0.4 \pm 0.5$ & $0.2 \pm 0.4$ & $0.2 \pm 0.4$ \\
\hline Polychaeta & Petitia amphophthalma & $1 \pm 2.2$ & 0 & 0 & 0 & 0 & 0 \\
\hline Polychaeta & Pionosyllis spinisetosa & 0 & $1.2 \pm 0.8$ & $1.4 \pm 2.1$ & $1.4 \pm 3.1$ & $0.2 \pm 0.4$ & 0 \\
\hline Polychaeta & Protodorvillea kefersteini & $0.2 \pm 0.4$ & 0 & 0 & 0 & 0 & 0 \\
\hline Polychaeta & Rhynchospio glutaea & $0.6 \pm 0.9$ & $0.2 \pm 0.4$ & $0.8 \pm 1.3$ & 0.8 & $1.6 \pm 2.1$ & $1.2 \pm 1.1$ \\
\hline Polychaeta & Schoeredella laubieri & $0.6 \pm 0.5$ & $1.2 \pm 0.8$ & 0 & 0 & $0.4 \pm 0.5$ & 0 \\
\hline Polychaeta & Scoloplos armiger & 0 & 0 & 0 & 0 & 0 & 0 \\
\hline Polychaeta & Sphaerosyllis xarifae & 0 & 0 & 0 & 0 & 0 & 0 \\
\hline Polychaeta & Spio decoratus & $0.2 \pm 0.4$ & 0 & 0 & 0 & 0 & 0 \\
\hline Polychaeta & Spio filicornis & $0.6 \pm 1.3$ & $4.6 \pm 2.2$ & $2.4 \pm 2.8$ & $7.6 \pm 3.8$ & $3.8 \pm 5.4$ & $4.2 \pm 1.6$ \\
\hline Polychaeta & Streptosyllis bidentata & $3.6 \pm 3.5$ & $0.6 \pm 0.5$ & 0 & $0.2 \pm 0.4$ & 0 & 0 \\
\hline Polychaeta & Streptosyllis campoyi & $0.6 \pm 0.9$ & 0 & 0 & 0 & 0 & $0.2 \pm 0.4$ \\
\hline Polychaeta & Syllides edentulus & 0 & 0 & $0.4 \pm 0.9$ & 0 & 0 & 0 \\
\hline Group & Species & $\begin{array}{c}\text { November } \\
2000\end{array}$ & $\begin{array}{c}\text { December } \\
2000\end{array}$ & $\begin{array}{c}\text { January } \\
2001\end{array}$ & $\begin{array}{c}\text { February } \\
2001\end{array}$ & $\begin{array}{c}\text { March } \\
2001\end{array}$ & $\begin{array}{l}\text { April } \\
2001\end{array}$ \\
\hline Polychaeta & Aricidea (Acmira) catherinae & 0 & 0 & 0 & $0.4 \pm 0.9$ & 0 & 0 \\
\hline Polychaeta & Brania arminii & 0 & 0 & 0 & 0 & 0 & 0 \\
\hline Polychaeta & Capitomastus minimus & $0.4 \pm 0.9$ & $0.4 \pm 0.5$ & $0.2 \pm 0.4$ & $0.2 \pm 0.4$ & 0 & 0 \\
\hline Polychaeta & Notomastus latericeus & 0 & 0 & 0 & 0 & 0 & 0 \\
\hline Polychaeta & Cauleriella bioculata & 0 & 0 & 0 & 0 & 0 & 0 \\
\hline Polychaeta & Cirriformia tentaculata & 0 & 0 & 0 & 0 & 0 & $0.2 \pm 0.4$ \\
\hline Polychaeta & Cirrophorus furcatus & $0.2 \pm 0.4$ & 0 & 0 & 0 & 0 & $0.6 \pm 0.9$ \\
\hline Polychaeta & Cirrophorus perdidoensis & 0 & 0 & 0 & 0 & 0 & 0 \\
\hline Polychaeta & Dispio uncinata & 0 & 0 & $0.2 \pm 0.4$ & 0 & 0 & 0 \\
\hline Polychaeta & Exogone breviantennata & 0 & 0 & 0 & 0 & 0 & 0 \\
\hline Polychaeta & Microphthalmus pseudoaberrans & 0 & $0.2 \pm 0.4$ & 0 & 0 & 0 & 0 \\
\hline Polychaeta & Ophelia bicornis & 0 & 0 & 0 & 0 & 0 & 0 \\
\hline Polychaeta & Protodrilus aff. rubropharyngeus & 0 & 0 & 0 & 0 & 0 & 0 \\
\hline Polychaeta & Parapionosyllis macaronesiensis & 0 & 0 & 0 & 0 & $0.2 \pm 0.4$ & 0 \\
\hline Polychaeta & Parapionosyllis minuta & 0 & 0 & 0 & 0 & 0 & 0 \\
\hline Polychaeta & Parapionosyllis n. sp. & 0 & 0 & 0 & 0 & 0 & $0.4 \pm 0.5$ \\
\hline Polychaeta & Perinereis cultrifera & $0.2 \pm 0.4$ & 0 & 0 & 0 & 0 & 0 \\
\hline Polychaeta & Periquesta canariensis & $0.8 \pm 1.3$ & $0.8 \pm 0.4$ & $0.2 \pm 0.4$ & $0.8 \pm 0.4$ & $0.4 \pm 0.5$ & $0.2 \pm 0.4$ \\
\hline Polychaeta & Petitia amphophthalma & 0 & $0.6 \pm 0.5$ & 0 & $0.2 \pm 0.4$ & 0 & 0 \\
\hline Polychaeta & Pionosyllis spinisetosa & $0.6 \pm 0.5$ & $0.2 \pm 0.4$ & $0.6 \pm 0.9$ & $1.8 \pm 3.5$ & $0.2 \pm 0.4$ & $0.4 \pm 0.5$ \\
\hline Polychaeta & Protodorvillea kefersteini & 0 & 0 & 0 & 0 & 0 & 0 \\
\hline Polychaeta & Rhynchospio glutaea & $1.6 \pm 1.1$ & $0.6 \pm 0.5$ & $1.8 \pm 1.3$ & $0.4 \pm 0.5$ & $3.4 \pm 0.5$ & $1.8 \pm 2.5$ \\
\hline Polychaeta & Schoeredella laubieri & $2 \pm 3.5$ & $1 \pm 0.7$ & 0 & $0.2 \pm 0.4$ & 0 & 0 \\
\hline Polychaeta & Scoloplos armiger & $0.4 \pm 0.9$ & 0 & 0 & 0 & 0 & 0 \\
\hline Polychaeta & Sphaerosyllis xarifae & $0.2 \pm 0.4$ & 0 & 0 & 0 & 0 & 0 \\
\hline Polychaeta & Spio decoratus & 0 & 0 & 0 & 0 & 0 & 0 \\
\hline Polychaeta & Spio filicornis & $9.4 \pm 7.8$ & $8.2 \pm 4.1$ & $7.4 \pm 3.8$ & $1.4 \pm 1.5$ & $10 \pm 4.6$ & $7.2 \pm 5.1$ \\
\hline Polychaeta & Streptosyllis bidentata & $0.2 \pm 0.4$ & 0 & $0.6 \pm 0.9$ & $0.4 \pm 0.5$ & 0 & 0 \\
\hline Polychaeta & Streptosyllis campoyi & $0.4 \pm 0.5$ & $1.8 \pm 2$ & $0.6 \pm 0.9$ & $0.6 \pm 0.9$ & 0 & 0 \\
\hline Polychaeta & Syllides edentulus & 0 & 0 & 0 & 0 & 0 & 0 \\
\hline
\end{tabular}


Continued from Table 2

\begin{tabular}{|c|c|c|c|c|c|c|c|}
\hline Group & Species & $\begin{array}{l}\text { May } \\
2000\end{array}$ & $\begin{array}{l}\text { June } \\
2000\end{array}$ & $\begin{array}{l}\text { July } \\
2000\end{array}$ & $\begin{array}{c}\text { August } \\
2000\end{array}$ & $\begin{array}{c}\text { September } \\
2000\end{array}$ & $\begin{array}{l}\text { October } \\
2000\end{array}$ \\
\hline Polychaeta & Syllides japonicus & $0.2 \pm 0.4$ & 0 & 0 & 0 & 0 & $1 \pm 1.4$ \\
\hline Polychaeta & Syllis armillaris & 0 & 0 & 0 & 0 & $0.2 \pm 0.4$ & 0 \\
\hline Polychaeta & Syllis garciai & 0 & 0 & $1.4 \pm 1.9$ & 0 & 0 & 0 \\
\hline Turbellaria & Acoela sp. 1 & 0 & 0 & $0.2 \pm 0.4$ & 0 & $0.2 \pm 0.4$ & $0.2 \pm 0.4$ \\
\hline Turbellaria & Catenulidasp. $1(L)$ & $1.8 \pm 1.5$ & 1 & $3.6 \pm 3.4$ & $5.4 \pm 4.8$ & $5 \pm 2.5$ & $2.6 \pm 0.5$ \\
\hline Turbellaria & Catenulidasp. 2 (S) & $0.2 \pm 0.4$ & $1.2 \pm 2.2$ & 0 & $1.6 \pm 1.1$ & $3.6 \pm 3.9$ & $0.6 \pm 0.5$ \\
\hline Turbellaria & Catenulida sp. 3 (M) & $3 \pm 3.5$ & $0.8 \pm 1.8$ & 0 & $0.4 \pm 0.5$ & $1.2 \pm 1.3$ & $1.8 \pm 2.4$ \\
\hline Turbellaria & Catenulida aff. catenulidae & $0.2 \pm 0.4$ & $0.2 \pm 0.4$ & 0 & 0 & $0.8 \pm 1.1$ & $2 \pm 1.2$ \\
\hline Turbellaria & Proseriatasp. 1 & $1 \pm 1.4$ & $0.2 \pm 0.4$ & 0 & $0.2 \pm 0.4$ & $1 \pm 1.2$ & 0 \\
\hline Turbellaria & Proseriatasp. 2 & 0 & $0.2 \pm 0.4$ & 0 & 0 & $1 \pm 1.2$ & 0 \\
\hline Turbellaria & Rhabdocoela sp. 1 & $0.2 \pm 0.4$ & 0 & 0 & 0 & $0.2 \pm 0.4$ & $0.4 \pm 0.5$ \\
\hline Oligochaeta & Aktedrilus sp. & $1.2 \pm 1.3$ & $23.6 \pm 35.2$ & $15.6 \pm 18.8$ & $1 \pm 1$ & $0.8 \pm 1.3$ & $0.8 \pm 1.3$ \\
\hline Oligochaeta & Coralliodrilus sp. & 0 & $1.4 \pm 2.6$ & $0.6 \pm 1.3$ & 0 & 0 & $0.2 \pm 0.4$ \\
\hline Oligochaeta & Enchytraeidae sp. & $0.2 \pm 0.4$ & $4.4 \pm 4.3$ & $3 \pm 4.2$ & $0.2 \pm 0.4$ & 0 & 0 \\
\hline Oligochaeta & Graniasp. & $1.2 \pm 1.8$ & $7.6 \pm 11.5$ & $3 \pm 4.1$ & $0.6 \pm 0.9$ & $0.4 \pm 0.9$ & $0.8 \pm 1.8$ \\
\hline Oligochaeta & Heterodrilus sp. & 0 & $11.2 \pm 21.3$ & $4 \pm 6.3$ & 0 & 0 & $0.2 \pm 0.4$ \\
\hline Oligochaeta & Heterodrilus sp. 1 & 0 & 0 & $1 \pm 1.4$ & 0 & 0 & 0 \\
\hline Oligochaeta & Tubificidae sp. & 0 & 0 & 0 & 0 & 0 & 0 \\
\hline Acari & Acaridae & 0 & 0 & 0 & 0 & 0 & $0.2 \pm 0.4$ \\
\hline Cnidaria & Halamnohydra sp. & $0.2 \pm 0.4$ & 0 & 0 & 0 & 0 & 0 \\
\hline Echinodermata & Echiuroidea & $0.2 \pm 0.4$ & 0 & 0 & 0 & 0 & 0 \\
\hline Amphipoda & Bathyporeia elegans & $0.2 \pm 0.4$ & 0 & $0.2 \pm 0.4$ & 0 & 0 & 0 \\
\hline Amphipoda & Lysianassidae & 0 & 0 & 0 & 0 & 0 & 0 \\
\hline Amphipoda & Pontocrates arenarius & 0 & 0 & 0 & 0 & 0 & $0.2 \pm 0.4$ \\
\hline Amphipoda & Urothoesp. & $0.2 \pm 0.4$ & 0 & 0 & 0 & 0 & 0 \\
\hline Decapoda & Callianassa tyrrhena & 0 & 0 & 0 & 0 & $0.2 \pm 0.4$ & 0 \\
\hline Nemertea & Ototyphlonemertessp. 1 & 0 & 0 & 0 & 0 & 0 & 0 \\
\hline Misidacea & Gastrosaccus sanctus & 0 & $0.6 \pm 0.9$ & 0 & 0 & 0 & 0 \\
\hline Ostracoda & Cypridina aff. mediterranea & 0 & 0 & $0.2 \pm 0.4$ & 0 & 0 & 0 \\
\hline Tanaidacea & Apseudes talpa & $0.8 \pm 1.1$ & 0 & 0 & 0 & 0 & $0.6 \pm 0.5$ \\
\hline Group & Species & $\begin{array}{c}\text { November } \\
2000\end{array}$ & $\begin{array}{c}\text { December } \\
2000\end{array}$ & $\begin{array}{c}\text { January } \\
2001\end{array}$ & $\begin{array}{c}\text { February } \\
2001\end{array}$ & $\begin{array}{c}\text { March } \\
2001\end{array}$ & $\begin{array}{l}\text { April } \\
2001\end{array}$ \\
\hline Polychaeta & Syllides japonicus & 0 & 0 & 0 & 0 & $0.4 \pm 0.5$ & $1.6 \pm 2.6$ \\
\hline Polychaeta & Syllis armillaris & 0 & 0 & 0 & 0 & 0 & $0.6 \pm 1.3$ \\
\hline Polychaeta & Syllis garciai & 0 & 0 & 0 & 0 & 0 & 0 \\
\hline Turbellaria & Acoelasp. 1 & $0.6 \pm 1.3$ & $0.8 \pm 0.8$ & $0.6 \pm 1.3$ & $0.4 \pm 0.5$ & 0 & 0 \\
\hline Turbellaria & Catenulidasp. $1(L)$ & $2.8 \pm 1.1$ & $2.6 \pm 2.2$ & $6.8 \pm 2.6$ & $1.8 \pm 1.6$ & 0 & 0 \\
\hline Turbellaria & Catenulidasp. 2 (S) & $0.4 \pm 0.5$ & $0.6 \pm 0.9$ & $1 \pm 1$ & $0.2 \pm 0.4$ & 0 & 0 \\
\hline Turbellaria & Catenulida sp. 3 (M) & 0 & $2.4 \pm 1.7$ & $1.2 \pm 1.8$ & 0 & 0 & 0 \\
\hline Turbellaria & Catenulida aff. catenulidae & $2 \pm 1.4$ & $2.6 \pm 2.7$ & $3.6 \pm 2.6$ & $0.2 \pm 0.4$ & 0 & 0 \\
\hline Turbellaria & Proseriatasp. 1 & $0.8 \pm 1.8$ & $1 \pm 1$ & $5.8 \pm 4.9$ & 2 & 0 & 0 \\
\hline Turbellaria & Proseriatasp. 2 & 0 & 0 & 0 & 0 & 0 & 0 \\
\hline Turbellaria & Rhabdocoela sp. 1 & 0 & 0 & $0.4 \pm 0.5$ & 0 & 0 & 0 \\
\hline Oligochaeta & Aktedrilus sp. & $2.4 \pm 4.3$ & $0.2 \pm 0.4$ & $0.4 \pm 0.5$ & $0.4 \pm 0.5$ & $5.8 \pm 11.9$ & $13 \pm 21.4$ \\
\hline Oligochaeta & Coralliodrilus sp. & 0 & 0 & 0 & 0 & 0 & $0.2 \pm 0.4$ \\
\hline Oligochaeta & Enchytraeidae sp. & $0.8 \pm 0.8$ & 0 & 0 & 0 & $0.4 \pm 0.9$ & $3.8 \pm 8.5$ \\
\hline Oligochaeta & Grania sp. & $3.8 \pm 6.5$ & $0.2 \pm 0.4$ & 0 & 0 & $3 \pm 6.7$ & $11.6 \pm 23.7$ \\
\hline Oligochaeta & Heterodrilus sp. & $0.2 \pm 0.4$ & 0 & 0 & 0 & $3.4 \pm 7.6$ & $0.8 \pm 1.1$ \\
\hline Oligochaeta & Heterodrilus sp. 1 & 0 & 0 & 0 & 0 & 0 & $0.2 \pm 0.4$ \\
\hline Oligochaeta & Tubificidae sp. & 0 & 0 & 0 & $0.4 \pm 0.5$ & $2.2 \pm 3.9$ & $0.4 \pm 0.9$ \\
\hline Acari & Acaridae & 0 & $0.2 \pm 0.4$ & 0 & 0 & 0 & 0 \\
\hline Cnidaria & Halamnohydra sp. & 0 & 0 & 0 & 0 & 0 & 0 \\
\hline Echinodermata & Echiuroidea & 0 & 0 & 0 & 0 & 0 & 0 \\
\hline Amphipoda & Bathyporeia elegans & 0 & 0 & $0.2 \pm 0.4$ & 0 & $0.2 \pm 0.4$ & 0 \\
\hline Amphipoda & Lysianassidae & $0.2 \pm 0.4$ & 0 & 0 & 0 & 0 & 0 \\
\hline Amphipoda & Pontocrates arenarius & $0.6 \pm 0.9$ & 0 & $0.4 \pm 0.9$ & $0.4 \pm 0.5$ & $0.6 \pm 1.3$ & 0 \\
\hline Amphipoda & Urothoesp. & 0 & 0 & 0 & 0 & 0 & 0 \\
\hline Decapoda & Callianassa tyrrhena & 0 & 0 & 0 & 0 & 0 & 0 \\
\hline Nemertea & Ototyphlonemertessp. 1 & 0 & 0 & 0 & $0.2 \pm 0.4$ & 0 & 0 \\
\hline Misidacea & Gastrosaccus sanctus & 0 & 0 & 0 & 0 & 0 & 0 \\
\hline Ostracoda & Cypridina aff. mediterranea & 0 & $0.2 \pm 0.4$ & 0 & 0 & $0.2 \pm 0.4$ & 0 \\
\hline Tanaidacea & Apseudes talpa & $2 \pm 2.1$ & $13.6 \pm 8.9$ & 0 & $0.8 \pm 0.8$ & 0 & 0 \\
\hline
\end{tabular}


Meiofauna density ranged from 151.63 ind./ $10 \mathrm{~cm}^{2}$ (May 2000) to 853.46 ind. $/ 10 \mathrm{~cm}^{2}$ (January 2001) (Fig. 2). Meiofaunal abundances were more or less stable throughout the study year, however, a sharp decrease occurred in May 2000, posteriorly recovered two months later (July 2000). As expected, meiofaunal abundance showed highly significant differences during the study period (one-way ANOVA, $F=17.612 ; p \ll 0.0001$ ) (Fig. 2, Table 3).

\subsection{Nematodes}

Nematode abundances ranged from 117.6 ind. $/ 10 \mathrm{~cm}^{2}$ (May 2000) to 800 ind. $/ 10 \mathrm{~cm}^{2}$ (January 2001). Nematode densities obtained highly significant differences throughout the sampling period (one-way ANOVA, $F=19.476, p \ll 0.001$ ) (Fig. 3, Table 3). A total of 74 species of free-living nematodes were identified. The most abundant species were Siphonolaimus sp. 2 (10 623 ind.) and Catanema sp. (8907 ind.), which made up ca. $50 \%$ of the overall meiofaunal abundance. Siphonolaimus sp. 2 abundances ranged from 3.4 ind. $/ 10 \mathrm{~cm}^{2}$ (May 2000) to 447 ind./ $10 \mathrm{~cm}^{2}$ (July 2000) (Fig. 4, Table 2). Catanema sp. abundan-

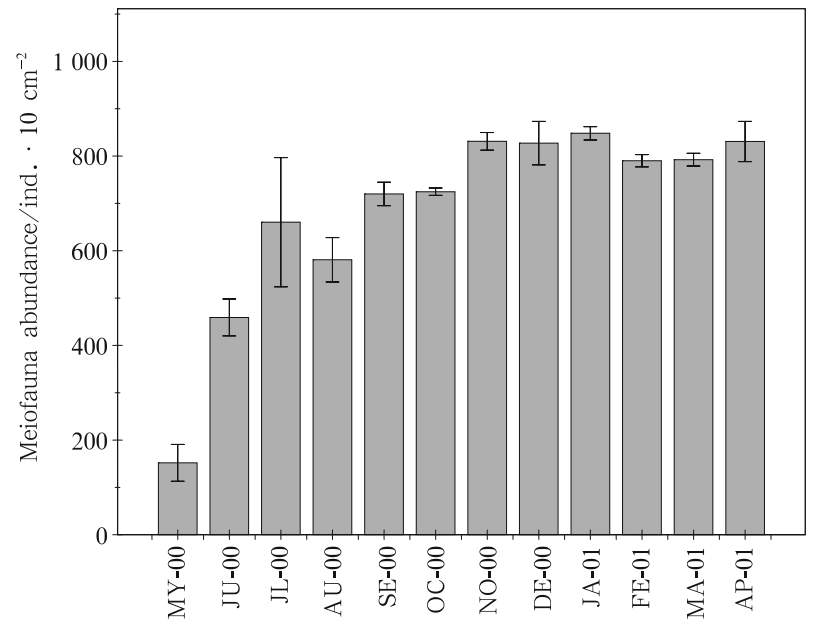

Fig.2. Meiofaunal abundances $( \pm \mathrm{SE})$ throughout the study period (May 2000-April 2001).

Table 3. Results of univariate ANOVA testing for differences in overall meiofaunal abundance and nematode, copepod, and oligochaete abundances throughout the study period (May 2000-April 2001)

\begin{tabular}{|c|c|c|c|c|c|c|c|c|c|}
\hline \multirow[t]{2}{*}{ Source of variation } & \multicolumn{3}{|c|}{$\begin{array}{l}\text { Overall meiofaunal } \\
\text { abundance }\end{array}$} & \multicolumn{3}{|c|}{$\begin{array}{l}\text { Nematode } \\
\text { abundance }\end{array}$} & \multicolumn{3}{|c|}{$\begin{array}{c}\text { Copepod } \\
\text { abundance }\end{array}$} \\
\hline & MS & $F$ & $p$ & MS & $F$ & $p$ & MS & $F$ & $p$ \\
\hline DF & 209750 & 17162 & $\ll 0.0001^{*}$ & 2289756 & 19476 & $\ll 0.0001^{*}$ & 440.05 & 4.238 & $0.0002^{*}$ \\
\hline \multirow[t]{2}{*}{ Source of variation } & \multicolumn{3}{|c|}{$\begin{array}{l}\text { Oligochaete } \\
\text { abundance }\end{array}$} & \multicolumn{3}{|c|}{$\begin{array}{c}\text { Siphonolaimus sp. } 2 \\
\text { abundance }\end{array}$} & \multicolumn{3}{|c|}{$\begin{array}{l}\text { Catanema sp. } \\
\text { bundance }\end{array}$} \\
\hline & MS & $F$ & $p$ & MS & $F$ & $p$ & MS & $F$ & $p$ \\
\hline DF & 1225.18 & 1.411 & 0.199 & 96464.37 & 6.103 & $\ll 0.0001^{*}$ & 36009 & 5.243 & $\ll 0.0001^{*}$ \\
\hline
\end{tabular}

Notes: * Significant differences $(p<0.01)$. MS represents mean squares and DF degrees of freedom.

ces ranged from 41.2 ind. $/ 10 \mathrm{~cm}^{2}$ (May 2000) to 334 ind. $/ 10 \mathrm{~cm}^{2}$ (February 2001) (Fig. 5, Table 2). Both species showed significant differences throughout the study period (Siphonolaimus sp. $2, F=6.10, p \ll 0.0001$; Catanema sp., $F=5.24, p \ll 0.000$ 1) (Table 3). In contrast, the species Ammotheristus sp., Enoplus sp., Rhabdodemania sp., and Tarvaia aff. peruvensis were scarce (Table 2).

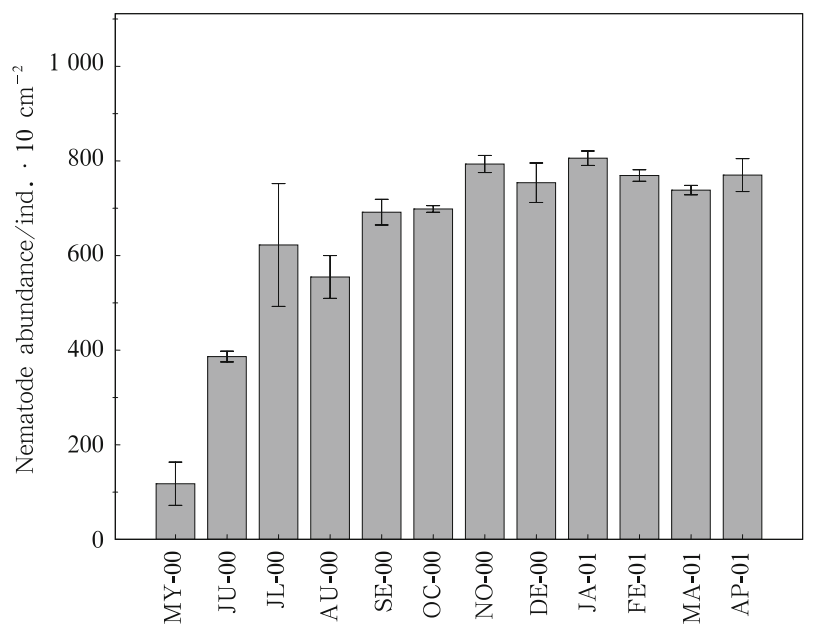

Fig.3. Nematode abundances $( \pm$ SE) throughout the study period (May 2000-April 2001).

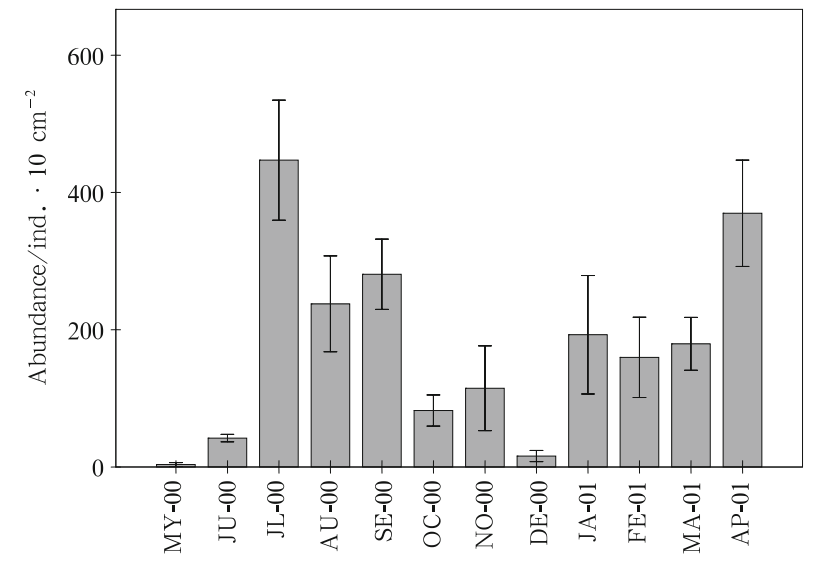

Fig.4. Nematode Siphonolaimus spB. Average abundances $( \pm$ SE) from May 2000 to April 2001.

\subsection{Oligochaetes}

Oligochaete abundances ranged from being less than 3 ind. $/ 10 \mathrm{~cm}^{2}$ (December 2000, January 2001, and February 2001) to 52 ind. $/ 10 \mathrm{~cm}^{2}$ (June 2000) (Fig. 4). This taxonomic group did not show significant differences in its abundance throughout the study period ( $F=1.411, p=0.199$ ) (Table 3). A total of seven oligochaete species were identified. The most abundant species were Aktedrilus sp. (326 ind.) and Grania sp. (161 ind.). In contrast, the species Coralliodrilus sp. (12 ind.) and Heterod- 


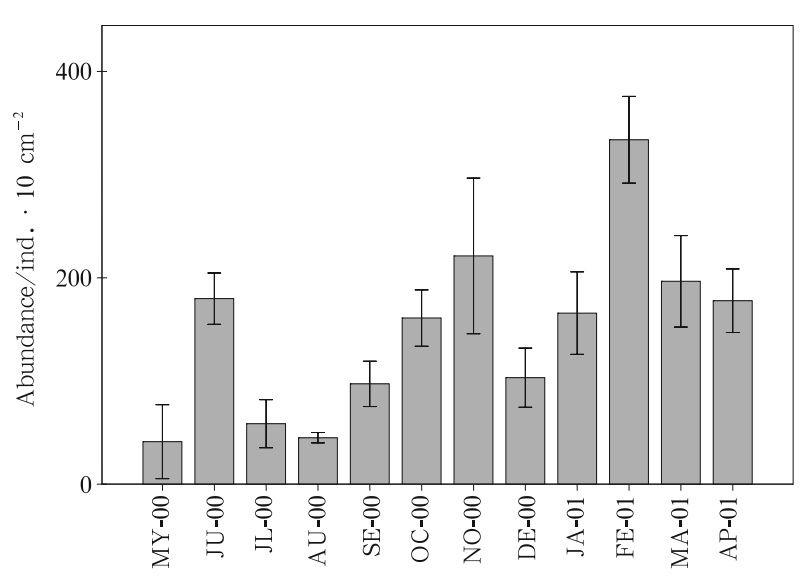

Fig.5. Nematode Catanema sp. Average abundances ( \pm SE) from May 2000 to April 2001.

rilus sp. 1 (6 ind.) were scarce (Table 2).

\subsection{Copepods}

Copepod abundances ranged from 1 ind./10 $\mathrm{cm}^{2}$ (July 2000) to 35 ind. $/ 10 \mathrm{~cm}^{2}$ (December 2000) (Fig. 6). Significant variations were found in copepod abundances during the study year ( $F=4.238 ; p=0.0002$ ) (Table 3 ). A total of nine copepod species were identified. The most abundant species were Halectinosoma sp. (255 ind.) and Harpacticus aff. flexus (163 ind.). In contrast, the taxa Tisbe aff. bulbisetosa, Asellopsis sp., and Paralaophonte sp. were scarce (Table 2).

\subsection{Polychaetes}

Polychaete abundances ranged from 7 ind./10 $\mathrm{cm}^{2}$ (February 2001) to 18 ind. $10 \mathrm{~cm}^{-2}$ (June 2000) (not shown). A total of 33 polychaete species were identified. The most abundant species was the spionid Spio filicornis (334 ind.). In contrast, 13 species were scarce (Table 2).

\subsection{Turbellarians}

Turbellarian abundances ranged from 3 ind. $/ 10 \mathrm{~cm}^{2}$ (June 2000 and July 2000) to 21 ind./10 $\mathrm{cm}^{2}$ (January 2001) (not shown). A total of eight turbellarian species were identified. The most abundant were Catenulida sp. 1 (198 ind.) and Proseriata sp. 1 (75 ind.). In contrast, the species Proseriata sp. 2 and Rhabdocoela sp. 1 were scarce (Table 2).

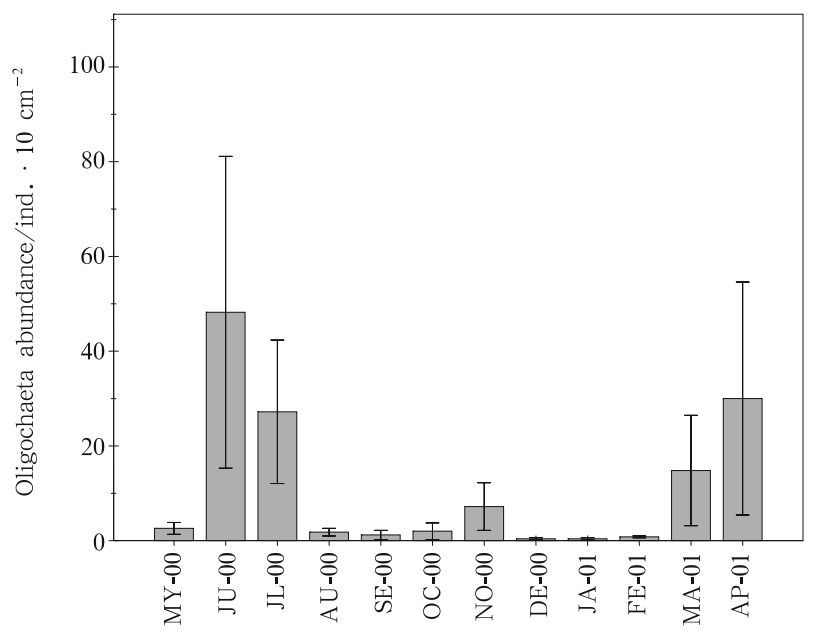

Fig.6. Oligochaeta. Average abundances $( \pm$ SE) from May 2000 to April 2001.

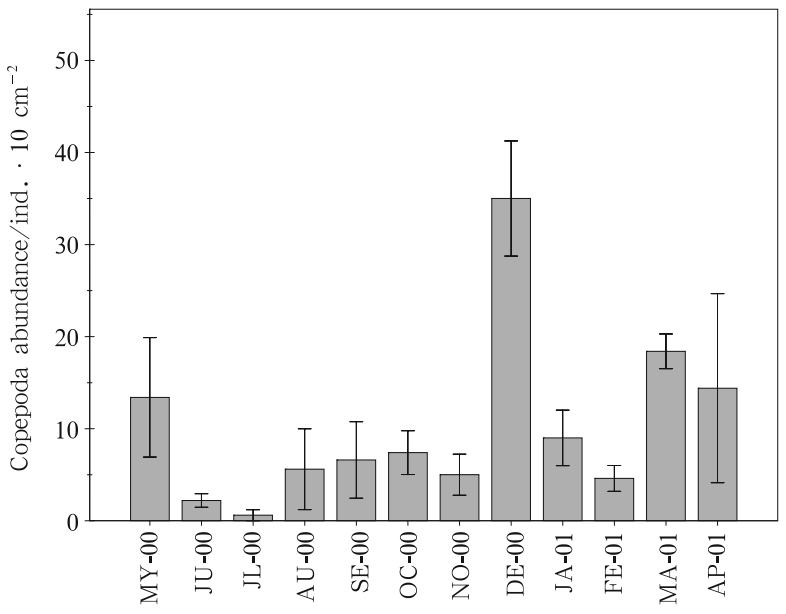

Fig.7. Copepoda. Average abundances $( \pm S E)$ from May 2000 to April 2001.

\subsection{Minor groups}

Minor taxa were constituted by acari, decapods, echinoids, amphipods, nemerteans, ostracods, kinorhynchs, and tanaids. A total of 13 species were identified, four of which were amphipods (Pontocrates arenarius, Bathyporeia elegans, Lysianassidae, and Urothoe sp.), while one species contributed to each of the remaining taxonomic groups. The most abundant species was the tanaid Apseudes talpa (89 ind.), followed by the amphipod Pontocrates arenarius (11 ind.). In contrast, seven species were scarce throughout the study period (Table 2).

\subsection{Multivariate analysis}

The cluster analysis using means of all months of the study year produced a dendrogram with six distinct groups (Fig. 8), which were also observed in the n-MDS (Fig. 9). The samples clustered in the dendrogram presented a high similarity $(>50 \%)$. Samples clustered in Group I corresponded to July 2000 (53\% of similarity), while those clustered in Group II corresponded to May 2000 (56\% of similarity). Samples from March and April 2001 were included in Group III (64\% of similarity) and those of June 2000 were included in Group IV (65\% of similarity). At the level of $69 \%$ of similarity, the remaining months of the year were separated into two groups (V and VI). Group V (August, September, October, and November 2000) and Group VI (December 2000, January 2001, and February 2001) were separated at the level of $70 \%$ of similarity. Group V was characterized by intermediate abundances of nematodes and Group VI obtained high densities of nematodes, polychaetes, and turbellarians.

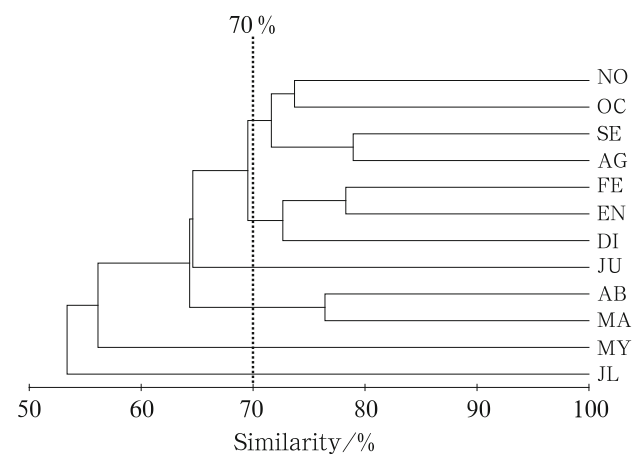

Fig.8. Dendrogram of similarity of sampling months during the study period (May 2000-April 2001). 


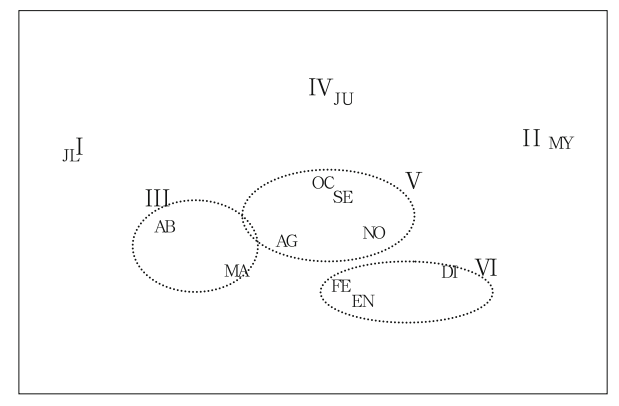

Fig.9. Multidimensional scaling (MDS) of sampling months during the study period (May 2000-April 2001) (stress $=0.14$ )

In terms of species composition, Group I (July 2000) was dominated by two nematode species belonging to the genera Siphonolaimus (sp. 1 and sp. 2) and Catanema sp. Group II (May 2000) was characterised by high densities of the nematodes Catanema sp., Oncholaimus campylocercoides, and the harpacticoid copepod Ectinosomatidae sp 1. Group III (March and April 2001) was dominated by the nematodes Siphonolaimus (sp. 1 and sp. 2) and Catanema sp. Group IV (June
2000) was overwhelmingly dominated by the nematode Catanemasp. and, to a lesser extent, by the nematodes Sabatieria celtica and Siphonolaimus sp. 2. Group V (August, September, October, and November 2000) were characterised by the nematodes Siphonolaimus sp. 2 and Catanema sp. Group VI (December 2000, January 2001, and February 2001) was dominated by the nematodes Catanema sp. and Siphonolaimus sp. 2, followed by Oncholaimus campylocercoides and Sabatieria celtica.

Meiofaunal assemblage structure showed temporal variations throughout the study period (May 2000-April 2001) (oneway ANOSIM, $R=0.249, p=0.003$ ). This result was mainly due to the temporal variability of the nematodes Siphonolaimus $\mathrm{sp}$. 2 and Catanema sp. that overwhelmingly dominated the meiofaunal assemblages. BIOENV analysis showed that variations in the percentages of organic matter, fine sands, and very fine sands clearly affected the meiofaunal assemblage structure of the sampling station ( $p=0.769$ ) (Table 4$)$. Thus, the former environmental variables, which showed temporal variations in the sampling location, were best for explaining meiofaunal abundances during the study period.

\section{Conclusions}

In the present study, we observed a response through time

Table 4. Summary of results from BIOENV analysis

\begin{tabular}{cl}
\hline Correlation & \multicolumn{1}{c}{ Variables } \\
\hline 0.769 & Organic matter, fine sands, and very fine sands \\
0.768 & Nitrogen, organic matter, fine sands, and very fine sands \\
0.752 & Organic matter, fine sands, very fine sands, and silt/clay \\
0.751 & Nitrogen, organic matter, fine sands, very fine sands, and silt/clay \\
0.735 & Fine sands and very fine sands \\
0.733 & Nitrogen, fine sands, and very fine sands \\
0.723 & Nitrogen, fine sands, very fine sands, and silt/clay \\
\hline
\end{tabular}

Notes: Faunal abundances were square root transformed and environmental variables were $\log _{10}(x+1)$ transformed.

at the assemblage level and major taxonomic groups, resulting in a temporal trend of meiofauna in the studied location. The trend was mainly caused by temporal fluctuations of the two most abundant species (nematodes Siphonolaimus sp. 2 and Catanema sp.), which dominated the meiofaunal assemblage during the study period. These variations were partially explained by temporal shifts of sedimentary variables (e.g., grain size composition and organic matter content).

Interstitial subtidal species are faced with two gradients: physical-horizontal (sediment stability) and chemical-vertical (oxygen concentration) gradients (McLachlan and Jaramillo, 1995; Armonies and Reise, 2000). The combination of those horizontal and vertical gradients may generate a complex spatial matrix of living conditions (Fenchel et al., 1967). In intertidal and subtidal seabeds, meiofaunal abundances vary according to temperature, tidal exposure, wave action, grain size, oxygen availability, substratum porosity, water percolation, organic input, and other factors. (Coull, 1988; Gheskiere et al., 2005). In general, nematodes dominate benthic meiofauna communities, comprising more than half of the overall meiofaunal abundances (McLachlan, 1983), as occurred in the present study. Harpacticoids are usually subdominant or a secondary group in terms of abundance (McIntyre, 1969).

The dominance of the nematodes Siphonolaimus sp. 2 and Catanema sp. was probably due to the trophic strategies of these species, which could be an advantage in oligotrophic environments, such as the study area (Canary Islands). Strong evidence has been found from the genus Catanema that it derives most of its carbon from autotrophic sulphur bacteria (Tom Moens pers. comm.). It is likely to get its energy from bacteria that act as endo- or ectosymbionts. In the case of endosymbionts, Catanema probably do not feed on them, although Catanema may digest part of them as they live in the intestine, but instead utilize dissolved secreted carbon sources from the bacteria. As ectosymbionts, Catanema could do the same (dissolved organic matter may penetrate through the cuticle) or they may feed on the bacteria growing on their cuticles (Tom Moens pers. comm.). However, a simpler explanation is that Catanema species may just feed on free-living bacteria, but that needs to be checked in future works. No detailed information about Siphonolaimus trophic strategies has been found in prior works.

In short, meiofaunal assemblages showed temporality in the sampling location throughout the study period. This temporal variation was partially explained by differences in the percentages of fine sands, very fine sands, and organic matter content throughout the study period. However, a detailed ecological study (including physical variables and biological interactions) is necessary in order to accurately explain temporal variations in this area. 


\section{Acknowledgements}

The authors are grateful to Dr. T Gheskiere and Dr. M Vincx (Department of Marine Biology, University of Ghent, Belgium) for bibliographic facilities and encouragement during the stay of the first author (R.R.). Thanks to Dr. T Moens (Department of Marine Biology, University of Ghent, Belgium), who provided autoecological data of free-living nematodes.

\section{References}

Armonies W, Reise K. 2000. Faunal diversity across a sandy shore. Mar Ecol Prog Ser, 196: 49-57

Brito M C, Núñez J, Martín D. 2005. Polychaetes associated to a $C y$ modocea nodosa meadow in the Canary Islands: assemblage structure, temporal variability and vertical distribution compared to other Mediterrranean seagrass meadows. Marine Biology, 146: 467-481

Boucher G. 1980. Facteurs d'équilibre d' un peuplement de Nématodes des sables sublittoraux. Mem Mus Nat Hist Nat N S Sér A Zool, 114: 1-181

Bradstreet R B. 1965. The Kjeldahl Method for Organic Nitrogen. Academic Press, 239

Buchanan J B. 1984. Sediment analysis. In: Holme N A, McIntyre A D, eds. Methods for the Study of Marine Benthos. 2nd ed. Oxford Blackwell Scientific Publications, 41-65

Clarke B. 1993. Non-parametric multivariate analyses of change in community structure. Aust J Ecol, 18: 117-143

Clarke K R, Gorley R N. 2001. PRIMER v5: User manual/tutorial. PRIMER-E. Plymouth, UK, 91

Coull B. 1988. Ecology of the marine meiofauna. In: Higgins R P, Thiel $\mathrm{H}$, eds. Introduction to The Study of Meiofauna. Washington DC: Smithsonian Institution Press, 18-38

Fenchel T, Jansson B O, Thun W. 1967. Vertical and horizontal distribution of the metazoan microfauna and of some physical factors in a sandy beach in the Northen part of the Oresund. Ophelia 42: $227-243$
Gheskiere T, Vincx M, Urban-Malinga B, et al. 2005. Nematodes from wave-dominated sandy beaches: diversity, zonation patterns and testing of the isocommunities concept. Est Coast Shelf Sci, 62: $365-375$

Higgins R P, Thiel H. 1988. Introduction o the study of meiofauna Washington DC: Smithsonian Institution Press

Juario J. 1975. Nematode species composition and seasonal fluctuation of a sublittoral meiofauna community in the German Bight. Veroff Inst Meeresforsch Bremerh, 15(4): 283-337

McIntyre A D. 1969. Ecology of marine meiobenthos. Biological Reviews, 44: 245-290

McLachlan A. 1983. Sandy beach ecology: a review. In: McLachlan A, Erasmus T, eds. Sandy Beaches as Ecosystems. The Hague: Junk, 321-330

McLachlan A, Jaramillo E. 1995. Zonation of sandy beaches. Oceanography and Marine Biology Annual Review, 33: 305-335

Ólafsson E, Elmgren R. 1997 Seasonaly dynamics of sublittoral meiobenthos in relation to seasonality in a coastal marine ecosystem. Est Coast Shelf Sci, 45: 149-164

Riera R. 2004. Meiofaunal biodiversity of Los Abrigos del Porís and Los Cristianos beaches. Assemblage structure and dynamics[dissertation]. Canary Islands, Spain: University of La Laguna

Rudnick D T, Elmgren R, Frithsen J B. 1985. Meiofaunal prominence and benthic seasonality in a coastal marine ecosystem. Oecologia, 67: 157-168

Short A D. 1999. Handbook of Beach and Shoreface Morphodynamics. New York: John Wiley and Sons

Somerfield P, Warwick R. 1996. Meiofauna in Marine Pollution Programmes. A laboratory manual. Lowestoft: Ministry of Agriculture, Fisheries, and Food, Directorate of Fisheries Research, 71

Warwick R, Buchanan J B. 1971. The meiofauna off coast of Northumberland: II. Seasonal variability of the nematode population. J Mar Biol Ass Uk, 51(1): 129-146 